Plant Tissue Cult. \& Biotech. 30(2): 307-313, 2020 (December)

CBangladesh Assoc. for Plant Tissue Culture \& Biotechnology

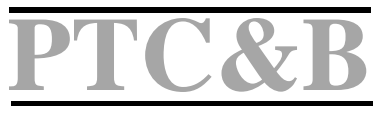

\title{
Phylogenetic Analysis of NHX1 Gene in Different Species of Plants Based on Coding Sequences
}

\author{
Tasmia Islam* \\ Department of Biochemistry and Molecular Biology, Jagannath University, Dhaka-1100, \\ Bangladesh
}

Keywords: Phylogenetic analysis, NHX1 gene, Coding sequence, Abiotic stress

The world population is anticipated to be about six billion by the end of the year 2050 which is very alarming (Najafi et al. 2019). On the other hand, food productivity is reducing due to various abiotic stresses; therefore, minimizing this situation is a major concern for all nations to cope with the increasing food requirements. Soil salinity is a serious environmental stress limiting plant productivity (Shrivastava et al. 2015). Sodium ions $\left(\mathrm{Na}^{+}\right)$abundant in saline soil, are cytotoxic in plants when they accumulate to high concentrations (Hasegawa et al. 2013). Over accumulation of $\mathrm{Na}^{+}$in the cytosol causes protein synthesis inhibition, many enzymatic reactions, and photosynthetic processes (Yamaguchi et al. 2013). Therefore, it is crucial to exclude $\mathrm{Na}^{+}$from photosynthetic organs for adequate metabolism and efficient carbon fixation.

Until now, several classes of $\mathrm{Na}^{+}$transporters have shown to play significant roles in $\mathrm{Na}^{+}$homeostasis during salinity stress (Uozumi et al. 2010). The NHX transporter has drawn particular attention because of its capability to transport $\mathrm{Na}^{+}$across cellular membranes. To date, most of NHX proteins studied facilitate electroneutral $\mathrm{Na}^{+} / \mathrm{H}^{+}$and $\mathrm{K}^{+} / \mathrm{H}^{+}$antiport through the membrane by using the $\mathrm{H}^{+}$gradient as a driving force (Uozumi et al. 2010). These were observed to be responsible for $\mathrm{Na}^{+}$reclusion into intracellular compartments under salinity stress. Overexpression of vacuolar NHX gene has conferred salt tolerance to a range of plant species such as wheat, rice, and tomato with a concomitant increase in tissue $\mathrm{Na}^{+}$(Sahoo et al. 2016, Zhang et al. 2017). Moreover, the role of vacuolar NHXs in $\mathrm{Na}^{+}$compartmentation under salinity stress is strongly supported by the findings of the Arabidopsis AtNHX1 mutant exhibited $\mathrm{Na}^{+}$sensitivity and significantly less vacuolar $\mathrm{Na}^{+} / \mathrm{H}^{+}$antiport activity (Apse et al. 2003). Besides an essential role in salt tolerance, $\mathrm{pH}$ regulation and organ developments have been demonstrated for vacuolar NHXs (Yamaguchi et al. 2001). Under normal conditions, the

*Author for correspondence: <tasmiaislam89@gmail.com>.

DOI: https://doi.org/10.3329/ptcb.v30i2.50700 
Arabidopsis AtNHX1/AtNHX2 double knockout plant showed severe plant growth defects, leaf cell expansion, and reproductive organ (Barragán et al. 2012, Bassil et al. 2011).

Plant genomes often contain several intracellular isoforms of the NHX gene (Bassilet al. 2012). These NHX transporters are classified into the IC-NHENHX family, a part of the large cation proton antiporter 1 (CPA1) family (Yamaguchi et al. 2013). IC-NHENHX family is further classified into vacuolar (Class I) or endosomal (Class II) NHXs, according to their sequence similarity and subcellular localizations of representative members (Bassil et al. 2012, Rodríguez-Rosales et al. 2009). Until now most of the plant species sequenced contain both types of NHXs (Bassil et al. 2012, Rodríguez-Rosales et al. 2009), and functional redundancies of vacuolar or endosomal NHXs had been recently reported in Arabidopsis (Bassil et al. 2011).

Recently, two distinct topology models have been suggested for AtNHX1. The protease protection analysis had been used to propose the first model in vacuoles of yeast expressing full-length AtNHX1 containing epitope tags inserted into each hydrophilic loop (Yamaguchi et al. 2003). This model is consist of nine transmembrane segments with a C-terminal hydrophilic tail facing toward the vacuolar lumen. There was another topology model was proposed on the basis of in vitro translation experiments using truncated AtNHX1 genes. The second model suggested that several transmembrane segments of AtNHX1 retain similar to pogenic properties as human NHE1 (Sato et al. 2005).

Phylogenetic analysis is a powerful tool for addressing many different evolutionary questions (Mansouri et al. 2013). Identifying of one gene and its homologs in several species indicates that these genes divergence from their common ancestor. The primary objective is to infer the pattern of the evolutionary relationships between the protein sequences of NHX1 gene that are being compared. These relationships will be revealed by the topology of the tree that will be constructed. This phylogeny will provide another perspective on biodiversity that allows an objective way to compare uniqueness and diversity of mentioned taxa.It is expected that our findings will shed light on functional characterization of mentioned NHX1 genes.

All sequences of NHX1 gene of different sources of organisms available in GenBank were downloaded from the NCBI database (www.ncbi.nlm.nih.gov/genbank), as showed in Table 1.

The program MEGA6 was used for multiple sequence alignment and phylogenetic tree construction. Multiple sequence alignment was done by the Clustal W method (http://www.genome.jp/tools/clustalw). The phylogenetic tree was drawn based on aligned protein sequences using the Maximum likelihood method based on the Poisson correction model (Tamura et al. 2013). The tree was drawn to scale, with branch lengths measured in the number of substitutions per site. 
Table 1. Descriptions of NHX1 gene sequences from different species.

\begin{tabular}{|c|c|c|c|c|c|c|}
\hline Accession No. & $\begin{array}{l}\text { cDNA } \\
\text { length } \\
\text { (bp) }\end{array}$ & $\begin{array}{l}\text { mRNA } \\
\text { length } \\
\text { (bp) }\end{array}$ & Protein Id & Family & Species & $\begin{array}{c}\text { No. of } \\
\text { sequence }\end{array}$ \\
\hline KX268353.1 & 1617 & 1617 & ANS57040.1 & Gramineae & $\begin{array}{l}\text { Hordeum vulgare } \\
\text { voucher }\end{array}$ & 1 \\
\hline KJ739865.1 & 1666 & 1666 & AJO67762.1 & Gramineae & Panicum virgatum & 2 \\
\hline AY131235.1 & 2668 & 2668 & AAN08157.1 & Amaranthaceae & Salicornia europaea & 3 \\
\hline AF507044.1 & 1955 & 1955 & AAQ07963.1 & Gramineae & $\begin{array}{l}\text { Lophopyrum } \\
\text { elongatum }\end{array}$ & 4 \\
\hline NM_122597.3 & 2553 & 2553 & NP_198067.1 & Brassicaceae & Arabidopsis thaliana & 5 \\
\hline NM_001250237.2 & 2732 & 2732 & NP_001237166.2 & Fabaceae & Glycine max & 6 \\
\hline KX132347.1 & 4370 & 4370 & AQN76250.1 & Salicaceae Mirb. & Populus pruinosa & 7 \\
\hline JF933902.1 & 1623 & 1623 & AEN03787.1 & Gramineae & Leptochloa fusca & 8 \\
\hline JX524227.1 & 2104 & 2104 & AFU88788.1 & Gramineae & Paeonia lactiflora & 9 \\
\hline KM083065.1 & 2313 & 2313 & AIQ86570.1 & Gramineae & Oryza sativa Indica & 10 \\
\hline AB982130.1 & 1629 & 1629 & BAP28595.1 & Convolvulaceae & Ipomoea tricolor & 11 \\
\hline AY607026.1 & 4920 & 4920 & AAT36679.1 & Rutaceae & Citrus Reticulata & 12 \\
\hline AY607025.1 & 4939 & 4939 & AAT36678.1 & Rutaceae & Citrus trifoliata & 13 \\
\hline KF941190.1 & 1602 & 1602 & AIA82923.1 & Gentianaceae & Gentiana rigescens & 14 \\
\hline GQ503257.1 & 2480 & 2480 & ADB80440.1 & Rosaceae & Malus zumi & 15 \\
\hline EU727217.1 & 1632 & 1632 & ACE78322.1 & Fabaceae & Lotus tenuis & 16 \\
\hline EU482408.2 & 1473 & 1473 & ACD64982.2 & Poaceae & Sorghum bicolor & 17 \\
\hline KX132304.1 & 4376 & 4376 & AQN76207.1 & Salicaceae & Populus euphratica & 18 \\
\hline КС608048.1 & 1668 & 1668 & AHC54576.1 & Amaranthaceae & Atriplex patens & 19 \\
\hline АJ306630.1 & 2122 & 2122 & CAC 84522.1 & Solanaceae & $\begin{array}{l}\text { Lycopersicon } \\
\text { esculentum }\end{array}$ & 20 \\
\hline KU156822.1 & 1587 & 1587 & ALP46546.1 & Papaveraceae & $\begin{array}{l}\text { Eschscholzia } \\
\text { californica }\end{array}$ & 21 \\
\hline KT306824.1 & 1946 & 1946 & ALT06549.1 & Iridaceae & Iris halophila & 22 \\
\hline EU109427.1 & 2394 & 2394 & ABV00895.1 & Fabaceae & Trifolium repens & 23 \\
\hline AB501188.1 & 2237 & 2237 & BAP90754.1 & Nelumbonaceae & Nelumbo nucifera & 24 \\
\hline KJ452342.1 & 1662 & 1662 & AHY19033.1 & Amaranthaceae & Suaeda pruinosa & 25 \\
\hline
\end{tabular}

A total of $25 \mathrm{cDNA}$ sequences of NHX1 genes from different source organisms were considered in silico analysis. According to (Table 1). Lengths of cDNA in 8 species like Hordeum vulgare voucher (1617 bp), Panicum virgatum (1666 bp), Leptochloa fusca (1623 bp), Ipomoea tricolor (1629 bp), Gentiana rigescens (1602 bp), Lotus tenuis (1632 bp), Atriplex patens (1668 bp), Suaeda pruinosa (1662 bp) were nearly equal that are belonging to the family Gramineae, Convolvulaceae, Gentianaceae, Fabaceae and Amaranthaceae 
respectively. Citrus Reticulata (4920 bp) and Citrus trifoliate (4939 bp) was also nearly equal in size belonging to the Rutaceae family. This feature showed that cDNA sequences of NHX1 genes in these species remain conserved during divergence from their common ancestor. Moreover, in all species, the length of mRNA and length of cDNA was equal, representing the NHX1 gene sequence is a whole exon region in this species. In silico analysis of NHX1 gene sequences and its comparison with its homologs in the other species, it revealed the sequence-based similarity between different species and clustering in distinct groups based on similarity.

Alignment by Clustal W Method: Multiple sequence alignment of NHX1 gene sequences showing homology in different regions (Fig. 1).

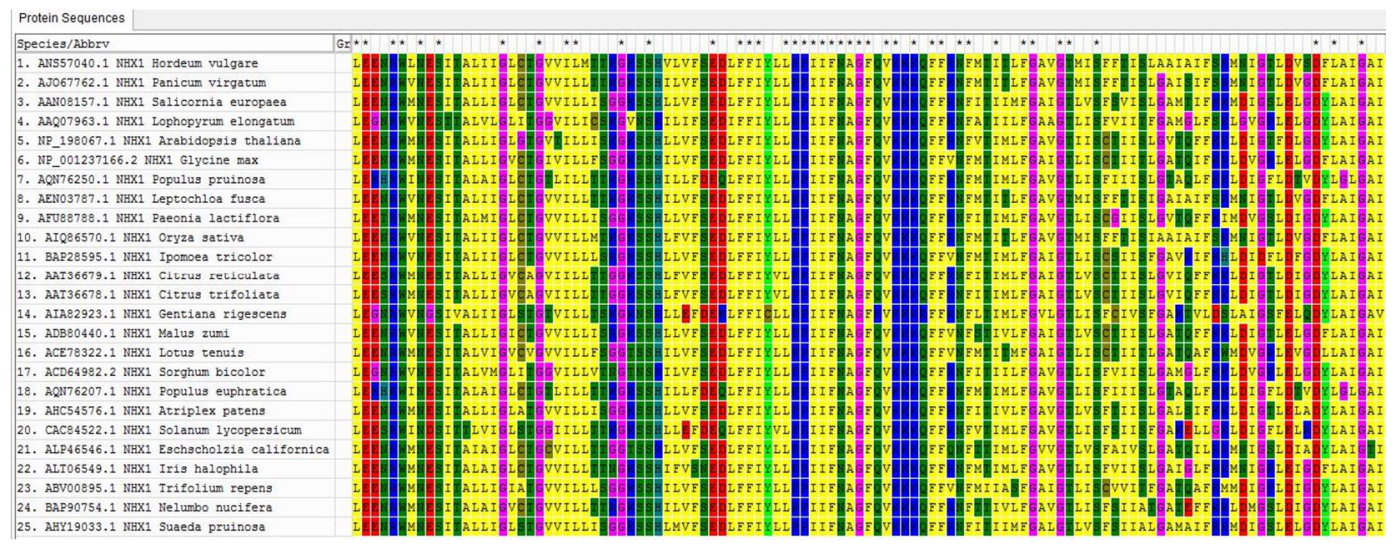

Fig. 1. Multiple sequence alignment of protein in a part of NHX1 gene sequence in the investigated species

The result of protein sequences alignment revealed that some sequences have high similarity for this gene and in some regions have a point different that can have an environmental effect such as mutation. Finally, the whole sequences' alignment showed the highly conserved region to primer design for PCR reaction to study genetic diversity and identification gene in plants.

Evolutionary divergence between sequences: The number of amino acid differences per site from between sequences were shown (Fig. 2). The analysis involved 25 amino acid sequences. All positions containing gaps and missing data were eliminated. There were a total of 399 positions in the final dataset.

Phylogenetic tree: This analysis showed evolutionary relationships predicted from the multiple sequence alignment. Each pair branches length's represents the distance between sequence pairs, and the units at the bottom of the tree show the number of substitution events. Results of the phylogenetic tree (Fig. 3) showed that species classified into four major groups, group 1 consists of 2 plant (Gentiana rigescens, Solanum lycopersicum); group 2 includes 2 plant (Populus pruinosa, Populus euphratica); group 3 consists of 1 plant (Eschscholzia californica); group 4 consists of 20 plants (Hordeum vulgare 
voucher, Panicum virgatum, Salicornia europaea, Lophopyrum elongatum, Arabidopsis thaliana, Glycine max, Leptochlo afusca, Paeoniala ctiflora, Oryza sativa Indica, Ipomoea tricolor, Citrus Reticulata, Citrus trifoliate, Maluszumi, Lotus tenuis, Sorghum bicolor, Atriplex patens, Iris halophile, Trifolium repens, Nelumbo nucifera, Suaeda pruinosa). The NHX1 sequence from Gentiana rigescens and Solanulym copersicum was assigned into the most distal clade.

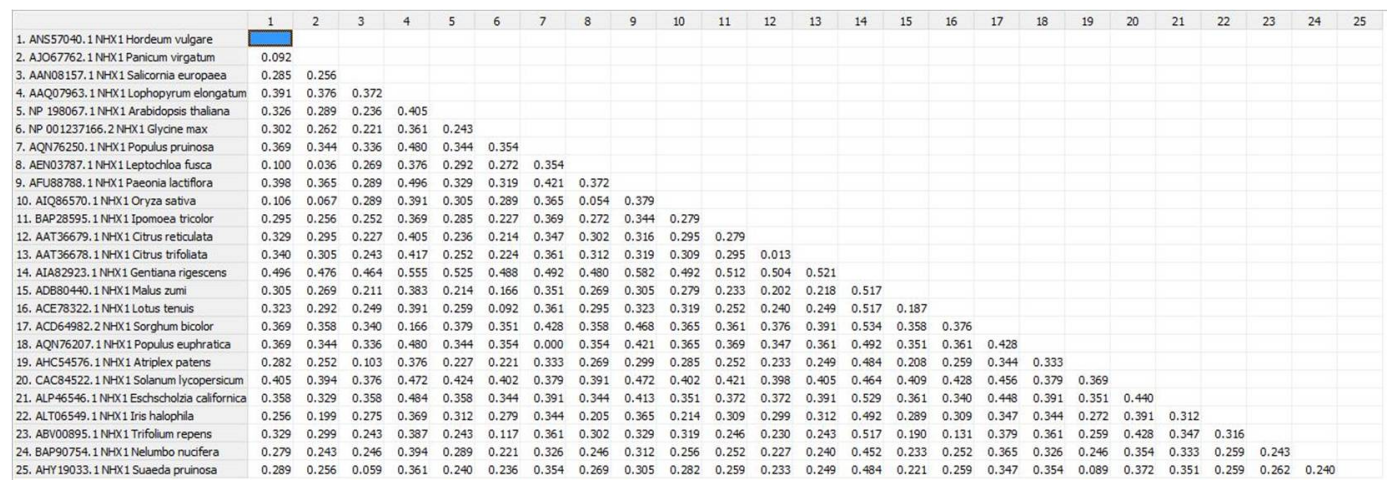

Fig. 2. E stimates of evolutionary divergence between sequences.

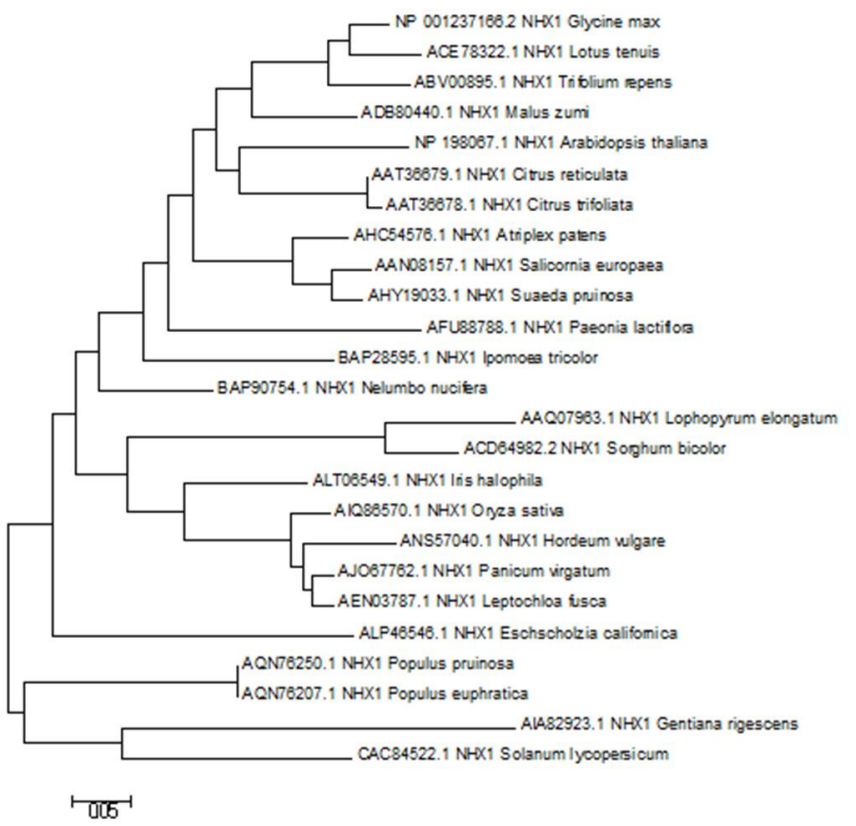

Fig. 3. Phylogenetic analysis of NHX1 gene identified in different source organisms.

The branching pattern in the phylogenetic tree (Fig. 3) reflects how species or other groups evolved from a series of common ancestors. This analysis suggested that plants' ancestor contained several NHX1 genes, which fashioned many regulatory proteins to 
control salt during abiotic stress. In recent studies, it has been found that compositional heterogeneity had an effect on the phylogenetic method (Sheffield 2012). Non stationary evolution (when the substitution pattern in nucleotide sequence is not uniform across an evolutionary tree) produces compositional heterogeneity (Sheffield 2012). Compositional heterogeneity was also observed in this study (Fig. 3). The Phylogenetic tree was constructed with NHX1 gene sequences from all species clarified the heterogeneous evolutionary lineages between these genes.

This study provides perspectives for future research on the NHX1 gene in diverse organisms, including substrate identities, physiological functions, and coevolution with NHX1 in their interacting partner organisms.

\section{References}

Apse MP, JB Sottosanto and E Blumwald (2003) Vacuolar cation/ $\mathrm{H}^{+}$exchange, ion homeostasis, and leaf development are altered in a T-DNA insertional mutant of AtNHX1, the Arabidopsis vacuolar $\mathrm{Na}^{+} / \mathrm{H}^{+}$antiporter. The Plant Journal 36: 229-239.

Barragán V, EO Leidi, Z Andrés, L Rubio, A De Luca, JA Fernández, B Cubero and JM Pardo (2012) Ion exchangers NHX1 and NHX2 mediate active potassium uptake into vacuoles to regulate cell turgor and stomatal function in Arabidopsis. The Plant Cell 24: 1127-1142.

Bassil E, H Tajima, Y-C Liang, M-a Ohto, K Ushijima, R Nakano, T Esumi, A Coku, M Belmonte and E Blumwald (2011) The Arabidopsis $\mathrm{Na}^{+} \mathbb{H}^{+}$antiporters NHX1 and NHX2 control vacuolar $\mathrm{pH}$ and $\mathrm{K}^{+}$homeostasis to regulate growth, flower development, and reproduction. The Plant Cell 23: 3482-3497.

Bassil E, A Coku and E Blumwald(2012) Cellular ion homeostasis: emerging roles of intracellular NHX Na${ }^{+} / \mathrm{H}^{+}$antiporters in plant growth and development. Journal of Experimental Botany 63: 5727-5740.

Dona MS, LA Prendergast, S Mathivanan, S Keerthikumar and A Salim (2017) Powerful differential expression analysis incorporating network topology for next-generation sequencing data. Bioinformatics 33: 1505-1513.

Tamura K, Stecher G, Peterson D, Filipski A, and Kumar S (2013) MEGA6: Molecular Evolutionary Genetics Analysis version 6.0. Molecular Biology and Evolution 30: 2725-2729.

Hasegawa and Paul M (2013) Sodium $\left(\mathrm{Na}^{+}\right)$homeostasis and salt tolerance of plants. Environmental and Experimental Botany 92: 19-31.

Mansouri S, AA Mehrabi and D Kahrizi (2013) Phylogenetic analysis of SOS1 gene in different species based on coding sequences. Middle-East J. Sci. 14: 1226-1229.

Najafi E and R Khanbilvardi (2019) Evaluating global crop distribution in the 21st century to maximize food production. AGUFM.2019: B31F-2440.

Rodríguez-Rosales MP, FJ Gálvez, R Huertas, MN Aranda, M Baghour, O Cagnac and K Venema(2009) Plant NHX cation/proton antiporters. Plant Signaling \& Behavior 4: 265-276.

Sahoo DP, S Kumar, S Mishra, Y Kobayashi, SK Panda and L Sahoo (2016) Enhanced salinity tolerance in transgenic mungbean overexpressing Arabidopsis antiporter (NHX1) gene. Molecular Breeding 36: 144. 
Sato $\mathbf{Y}$ and M Sakaguchi (2005) Topogenic properties of transmembrane segments of Arabidopsis thaliana NHX1 reveal a common topology model of the $\mathrm{Na}^{+} / \mathrm{H}^{+}$exchanger family. Journal of biochemistry. 138: 425-431.

Sheffield NC (2012) The interaction between base compositional heterogeneity and among-site rate variation in models of molecular evolution. ISRN Evolutionary Biology. 2013: 391561.

Shrivastava P and R Kumar (2015) Soil salinity: a serious environmental issue and plant growth promoting bacteria as one of the tools for its alleviation. Saudi Journal of Biological Sciences. 22: 123-131.

Uozumi Nobuyuki and Schroeder Julian I (2010) Ion channels and plant stress: past, present, and future. In: Ion Channels and Plant Stress Responses, Signaling and Communication in Plants, V. Demidchik and F. Maathuis (Eds.), Springer-Verlag, Berlin, pp. 1-22.

Yamaguchi T, S Fukada-Tanaka, Y Inagaki, N Saito, K Yonekura-Sakakibara, Y Tanaka, T Kusumi and S Iida (2001) Genes encoding the vacuolar $\mathrm{Na}^{+} \mathrm{H}^{+}$exchanger and flower coloration. Plant and Cell Physiology 42: 451-461.

Yamaguchi T, MP Apse, H Shi and E Blumwald (2003) Topological analysis of a plant vacuolar $\mathrm{Na}^{+} / \mathrm{H}^{+}$antiporter reveals a luminal $\mathrm{C}$ terminus that regulates antiportercation selectivity. Proceedings of the National Academy of Sciences 100: 12510-12515.

Yamaguchi T, S Hamamoto and N Uozumi (2013) Sodium transport system in plant cells. Frontiers in plant science. 4: 410.

Zhang W-D, P Wang, Z Bao, Q Ma, L-J Duan, A-K Bao, J-L Zhang and S-M Wang (2017) SOS1, HKT1; 5, and NHX1 synergistically modulate $\mathrm{Na}^{+}$homeostasis in the halophytic grass Puccinellia tenuiflora. Frontiers in plant science. 8: 576. 